\title{
Local structural excitations in model glass systems under applied load
}

\author{
S. Swayamjyoti, ${ }^{1}$ J. F. Löffler, ${ }^{2}$ and P. M. Derlet ${ }^{1, *}$ \\ ${ }^{1}$ Condensed Matter Theory Group, Paul Scherrer Institut, CH-5232 Villigen PSI, Switzerland \\ ${ }^{2}$ Laboratory of Metal Physics and Technology, Department of Materials, ETH Zurich, 8093 Zurich, Switzerland
}

(Received 22 January 2016; revised manuscript received 3 March 2016; published 6 April 2016)

\begin{abstract}
The potential-energy landscape of a model binary Lennard-Jones structural glass is investigated as a function of applied external strain, in terms of how local structural excitations (LSEs) respond to the load. Using the activation relaxation technique and nudged elastic band methods, the evolving structure and barrier energy of such LSEs are studied in detail. For the case of a tensile/compressive strain, the LSE barrier energies generally decrease/increase, whereas under pure shear, it may either increase or decrease resulting in a broadening of the barrier energy distribution. It is found that how a particular LSE responds to an applied strain is strongly controlled by the LSE's far-field internal stress signature prior to loading.
\end{abstract}

DOI: 10.1103/PhysRevB.93.144202

\section{INTRODUCTION}

Collective atomic-scale structural excitations mitigate the macroscopic plastic deformation of bulk metallic glasses (BMGs) [1]. In the work of Spaepen [2], a free-volume model was proposed which involves single-atom migration into regions of excess free volume. Argon, in subsequent work [3], introduced the notion of shear transformations (STs) where a finite number of atoms undergoes a local structural transformation to relieve stress. Both of these works relied on the concept of thermal activation. In 1998, the phenomenological concept of shear transformation zones (STZs) was postulated by Falk and Langer, and supported by high-strain-rate molecular dynamics simulations [4]. Subsequent high-strain-rate atomistic simulations gave insight into how collective STZ activity could lead to macroscopic plasticity [5-8].

Such molecular dynamics simulations probed predominantly athermal plasticity, in which the atomistic system is driven by an external load to a local material instability. A thermal-activation view of plasticity is, however, quite different. Here, thermal fluctuations allow the material to naturally undergo spontaneous structural excitations, allowing the system to explore its complex potential energy landscape (PEL) - even under zero load. In this context, the application of a load biases those structural transitions, whose stress signature is compatible with the applied load, to be more frequent and thus a net plasticity results. Evidence for thermally activated plasticity in BMGs is numerous for the case of high-temperature homogeneous plasticity [1] and also for lower-temperature heterogeneous plasticity, which involves the nucleation, propagation, and arrest of shear bands [9-12].

Computational methods exist to explore the PEL of computer-generated model glass systems without driving the system to an instability. A commonly used technique is the activation relaxation technique nouveau (ART $n$ ) [13-15]. ART $n$ begins in a local minimum of a PEL and climbs up the surrounding potential-energy hill to eventually converge to a saddle-point configuration through which the system must pass to enter a new neighboring local minimum configuration

\footnotetext{
*peter.derlet@psi.ch
}

of the PEL. This technique has been used in the past to study localized structural excitations in two-dimensional (2D) and 3D model glass systems [16-22]. In Ref. [20], the corresponding structural excitations have been referred to as local structural excitations (LSEs), which will be the terminology used in the present work.

In the work of Rodney and Schuh [16,17], a distribution of barrier energies that peaks at a finite value was found for model Lennard-Jones amorphous systems. If the computer-generated sample is relaxed well enough, this distribution appears to converge to zero as the barrier energy converges to zero. The authors also found little correlation between barrier energy and the corresponding shear-stress signature of the LSEs, suggesting a highly nontrivial PEL structure. Such a complexity is also reflected in a weak correlation between barrier energy and prefactor (which probes PEL curvature), derived from harmonic transition state theory [19]. The work of Ref. [20] investigated the characteristic atomic structure of the LSEs found by ARTn in terms of local pressure, volume, energy, bulk modulus, and eigenshear moduli. It revealed that smaller and more weakly bonded atoms are preferentially involved in LSEs identified by ARTn, which often occurred near regions of excess free volume. Using atomic-scale visualization techniques, the LSEs were seen to have an extended chainlike structure involving several atoms with a displacement generally comparable to the interatomic distance.

In the work of Fan et al. [21,22], ART $n$ was used to study the statistics of saddle-point configurations in a model $\mathrm{CuZr}$ system as a function of quench rate. The authors found their structural excitations were dominated by atoms, referred to as trigger atoms, whose displacements were maximal when compared to all atoms involved. The approximate relationship between these atomic displacements and their corresponding barrier energy was identified to be parabolic, giving a direct link to the construct of the Eshelby inclusion scenario [3,23]. In addition, a linear/parabolic correlation between the change in local von Mises stress and the corresponding atomic displacement/barrier energy was found. Strong deviations from the linear correlation between the von Mises stress and atomic displacements occurred mainly for the aforementioned trigger atoms. For less relaxed samples, the displacement of the trigger atoms was somewhat reduced, suggesting that the 
density of local minima in the glassy PEL increases. Indeed, the authors found this had consequences for the final end state of the structural excitation, where for the more unrelaxed systems the resulting plasticity was cascadelike rather than localized and involved a larger number of atoms than that of the trigger atoms [22].

In the present work, the ARTn method is applied to a computer-generated Lennard-Jones binary glass sample which was incrementally strained using several loading geometriesan imposed isotropic tensile and compressive strain, and pure shear strains in the $X Y, Y Z$, and $Z X$ planes. How the distribution of barrier energies is modified as a function of strain magnitude is studied. Individual LSEs are also studied as a function of strain via a combined ART $n$ and nudged elastic band (NEB) method, the latter of which allows for the determination of the minimum-energy path between two locally stable configurations. Section II details the methodology of ARTn and NEB to investigate LSEs under strain, and also introduces the concept of activation volume to characterize the stress dependence of LSE barrier energies. Section III constitutes the major findings and details the statistics of LSE barrier energies under tensile, compressive, and shear strains. Here, a number of LSEs are visualized at the atomic level to gain insight into how individual LSEs respond to an external load. Section IV discusses these results in terms of which features of an LSE under zero load might determine its barrier energy response to an external load, and illustrates more generally the implications of the current findings for early-stage plasticity (prior to shear banding).

\section{METHODOLOGY}

\section{A. Sample preparation}

The three-dimensional model 50:50 binary glass samples used in the present work are those used in Ref. [20], and consist of 1728 atoms interacting via the Wahnstrøm parametrized Lennard-Jones pair potential [24]. For this potential, the parameter $\varepsilon$ sets the energy scale and the parameter $\sigma_{11}$ sets the length scale. The computer-generated sample preparation procedure involves three steps: (1) equilibration of the liquid state using $N-P-T$ molecular dynamics at a constant temperature and hydrostatic pressure, (2) slow quenching of the sample from this well-equilibrated liquid to zero pressure and negligible temperature, and, finally, (3) molecular statics relaxation of the sample to a zero Kelvin local minimum. An atomic-scale structural analysis of the final sample is given in Ref. [20]. This past work also demonstrated that the results of the ARTn simulations do not depend on the particular realization of the computer-generated amorphous configuration.

\section{B. Loading conditions and strain control}

LSEs are investigated under tensile, compressive, and pure shear loading conditions via a static distortion of the simulation cell. For the tensile loading geometry, fractional volume changes of $0.00525,0.0105,0.01575$, and 0.021 are considered where upon each volume change the amorphous structure is relaxed. Similar negative volume changes are applied to investigate the compressive loading geometry.
A pure shear loading is applied by imposing the LeeEdwards boundary conditions [25] on the simulation cell. The samples are sheared to incremental shear-strain values of $\pm 2 \gamma=0.003,0.006,0.009$. 0.012, 0.015, 0.018, 0.021, $0.024,0.027$, and 0.03 . The $X Y, Y Z$, and $Z X$ shear planes are considered. The Lee-Edwards procedure imposes a shear strain by the use of a shifted boundary condition. For example, for an $X Y$ shear strain, atomic pairs crossing the periodic boundary along the $y$ axis are shifted along the $x$ axis by an amount equal to $-\gamma L_{y}$. Here, $\gamma$ is the desired shear strain and $L_{y}$ is the box length along the $y$ axis.

\section{ART $n$ and NEB investigations}

The ARTn [13-15] technique is employed in the present work to obtain multiple LSE barriers in strained glass samples. Starting from a local equilibrium configuration, ART $n$ converges to nearby saddle-point configurations of the PEL. The local minimum in which the glass system initially resides in is referred to as the "initial state." A saddle point directly connected to the initial state without any intermediary stable state in the path is referred to as the "activated state" corresponding to that local minimum.

To find a saddle-point configuration, ART $n$ begins by moving the configuration a certain distance along a randomly chosen $3 N$-dimensional direction. Here, this direction is chosen by first randomly selecting an atom and then randomly moving it a distance $0.15 \sigma_{11}$. The configuration is then moved along the eigenvector with the lowest eigenvalue in steps of $0.025 \sigma_{11}$ until the latter is less than $-3.0 \varepsilon / \sigma_{11}^{2}$. At each stage, the forces perpendicular to the eigenvector are relaxed. The above procedure is continued now using a variable step size of $\mathbf{F} \cdot \mathbf{u}_{0} / e_{0}$, where $\mathbf{F}$ is the current $3 N$-dimensional atomic force vector and $\mathbf{u}_{0}$ is the $3 N$-dimensional eigenvector corresponding to the lowest eigenvalue $e_{0}$. This step size is capped at $0.125 \sigma_{11}$. This is repeated until the maximum force on all atoms is less than $10^{-3} \varepsilon / \sigma_{11}$.

Upon convergence to the saddle point, the configuration is moved a little further in the direction of the eigenvector and subsequently relaxed via molecular statics to allow the configuration to fall into another local minimum. This local minimum configuration is referred to as the "final state," thereby defining an LSE by three atomic configurations, i.e., the initial state, the activated state, and the final state. The barrier energy of a particular LSE is then the difference in energy between the initial and activated state. To avoid redundant saddle-point configurations, converged activated states whose barrier energy differs by less than $0.001 \varepsilon$ from any other activated state are further inspected. If the center of positions of the LSEs (derived from the $3 N$-dimensional atomic displacement vectors between the initial and activated state) is less than $0.001 \sigma_{11}$, then the configuration is discarded.

To investigate how an individual LSE is modified by a loading geometry, the NEB method [26-30] is first used. This method requires the initial-state and the final-state configurations of an LSE. From these two configurations, the NEB method constructs the minimum-energy path (MEP), which is the lowest-energy path connecting the initial and the final state of an LSE. To do this, several intermediate configurations (known as images) along the path are constructed 
and connected via a harmonic spring model. Here, the initial images are constructed via a linear extrapolation between the initial and final states, and uniformly spaced along the MEP. To obtain a good estimate of the maximum energy along the MEP, 19 images are used with a spring constant equal to 100 $\varepsilon / \sigma_{11}^{2}$. The maximum energy configuration is then used as a starting point for ART $n$ to obtain an accurate saddle point. To ensure that the obtained saddle point is related to the zero-load saddle point, both the barrier energy and curvature are followed as a function of strain. If these quantities vary abruptly with strain, the associated LSE is no longer considered. Thus, by incrementally straining both the initial and final states via the methods outlined in Sec. II B, the corresponding strained MEPs can be obtained and the LSEs' structure as a function of loading can be followed.

\section{Activation volume}

Historically, the stress dependence of a thermally activated plastic process has been understood in terms of a stressdependent barrier energy [1,31]. The simplest parametrization of such a dependence is obtained by assuming that the barrier energy is a linear function of the applied stress $\tau$,

$$
E(\tau)=E(\tau=0)-\Omega \tau,
$$

where $\Omega$ has the unit of a volume. This latter term is referred to as the activation volume and is an important material parameter for thermally driven plasticity. For an isotropic applied strain, the appropriate $\tau$ is a hydrostatic pressure and $\Omega$ is always positive, reflecting the fact that barrier energies decrease/increase upon material dilation/compression. For the case of a pure shear strain, it will be shown that a barrier energy may either decrease or increase and thus the parameter $\Omega$ can be either negative or positive. In the present work, Eq. (1) will be fitted to obtain order-of-magnitude estimates of $\Omega$ for LSEs under the considered loading geometries.

\section{RESULTS}

\section{A. Elastic loading of samples}

For the straining geometries and associated strain magnitudes, the corresponding global stress tensor $\tau^{\mu \nu}$ can be calculated via

$$
\tau^{\mu \nu}=\frac{1}{2 V} \sum_{i j} \phi^{\prime}\left(R_{i j}\right) \frac{R_{i j}^{\mu} R_{i j}^{v}}{R_{i j}},
$$

which is valid for a pair interaction $\phi(r)$ such as the LennardJones potential. In the above equation, $V$ is the volume of the simulation cell and $R_{i j}$ is the interatomic distance between atoms $i$ and $j$. The gradient with respect to the applied strain of the global stress evaluated at zero strain will give the corresponding moduli. Such a curve is determined by appropriately straining the system, where at each strain increment the atomic configuration is relaxed to a zero-force state. With respect to an isotropic tensile/compressive strain, the resulting bulk modulus is found to equal $75.8 \epsilon /\left(\sigma_{11}\right)^{3}$, whereas the shear moduli for the $X Y, Y Z$, and $Z X$ geometries are found to equal $21.8,21.1$, and $20.5 \epsilon /\left(\sigma_{11}\right)^{3}$, respectively. These results compare well with the values found in past work [32].
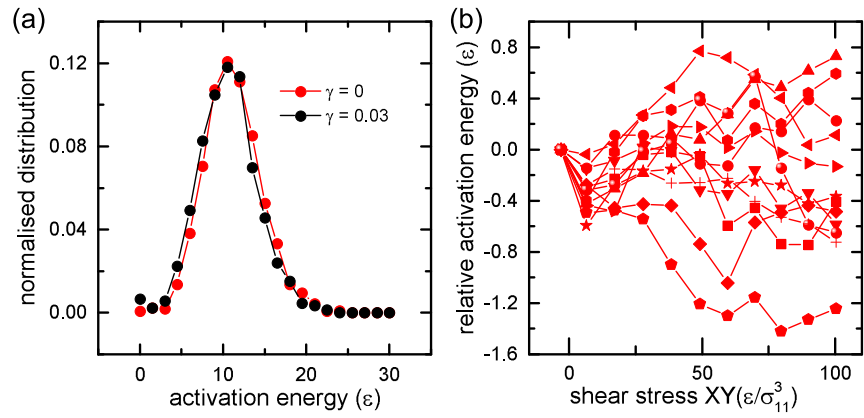

FIG. 1. (a) Normalized distributions of barrier energies obtained via ART $n$ for zero load and the maximum considered shear strain of $\gamma=0.03$. (b) Barrier-energy evolution as a function of $X Y$ shear-stress magnitude ( $\gamma \times$ appropriate shear modulus) for several individual LSEs derived via a combination of ART $n$ and NEB.

\section{B. Activation energy as a function of elastic strain}

In Fig. 1(a), the normalized distributions of the barrier energies obtained by ARTn are displayed for zero load and the maximum shear-strain magnitude considered. The data from the latter are the average obtained from the individual distributions for the considered $X Y, Y Z$, and $Z X$ shear strains. The distributions are derived from several thousand saddle points obtained from ART $n$. The figure reveals that the overall distribution changes little as a function of applied shear strain.

Detailed inspection of the data does, however, reveal a weak broadening and downward shift of the distribution by a scale less than $\varepsilon$ [this is seen in Fig. 3(a), which plots the mean of the obtained barrier energies]. The distribution broadening is evidenced by an increase in the ratio of the standard deviation to the average value as a function of increasing shear strain (not shown). The origin of this trend can be seen by inspecting how barrier energies of individual LSEs change with shear strain by using the combined ARTn and NEB approach outlined in Sec. II C. Figure 1(b) displays a plot of the change in barrier energy versus shear strain applied in the $X Y$ plane for a number of representative LSEs. The figure demonstrates that a given LSE's barrier energy can either increase or decrease by up to an order of $\varepsilon$. Similar trends are seen for the $Y Z$ and $Z X$ loading geometries.

The picture which therefore emerges is that under pure shear-strain loading geometry, a particular barrier energy may either increase or decrease. This is further confirmed by performing a similar NEB/ART $n$ analysis for several hundred LSEs. It is found (not shown) that the distribution of change in barrier energy, as a function of shear-strain magnitude, is distributed over positive and negative values, but biased towards a decrease in the barrier energy. This trend will be later quantified through investigating the distribution of resulting $\Omega$ 's [Eq. (1)].

In Fig. 1(a), a small peak in the low-barrier-energy regime is seen to emerge as a function of increasing strain magnitude. This occurs for the $X Y$ and $Z X$ shear geometries. Inspection of the structure of the saddle-point configurations reveals that they arise from LSEs involving the same single atom. Thus, in this case, ARTn converges a disproportional amount of times to the same LSE, but with a slightly different saddle-point configuration, indicating a very broad and low-barrier-energy 

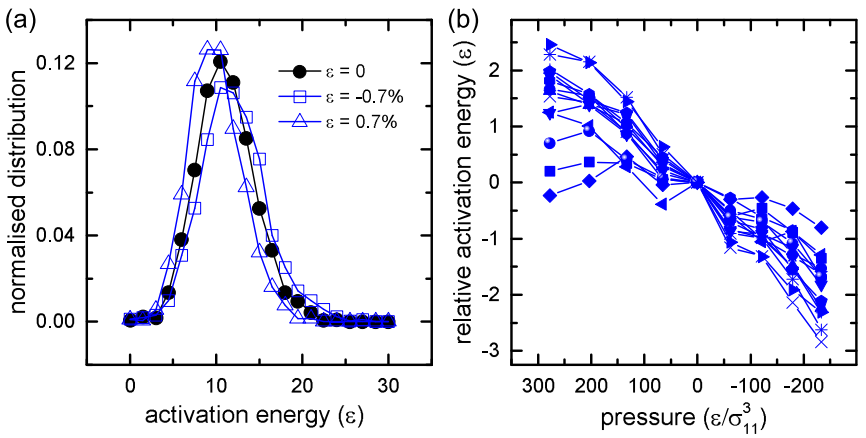

FIG. 2. (a) Normalized distribution of barrier energies obtained via $\mathrm{ART} n$ for zero load and an isotropic strain of $\pm 0.7 \%$. The distribution for the zero-load sample is also included. (b) Barrierenergy evolution as a function of isotropic stress magnitude $(\varepsilon \times$ bulk modulus) for several individual LSEs derived via a combination of $\mathrm{ART} n$ and NEB.

saddle-point structure. This may be seen as a very soft region of the material which responds only to the $X Y$ and $Z X$ applied shear strains. It is noted that the mean statistics shown in Fig. 3(a) do not include these LSEs.

Figure 2(a) shows the barrier-energy distributions as a function of both compressive and tensile isotropic strain. Again, the overall distributions do not greatly change as a function of strain. Closer inspection reveals a shift of the distribution to higher (compression) and lower (tension) barrier energies. There is also a weak broadening in both cases. This trend is quantified in Fig. 3(a), which shows the mean as a function of volume strain. The origin of this trend is again seen by inspecting how the barrier energy changes as a function of strain for individual LSEs. Figure 2(b) plots such data for some representative LSEs, where for compressive/tensile loadings, the LSE barrier energies generally increase/decrease.

Figure 3(b) shows the distribution of $\Omega$ estimates derived from the evolution of individual LSEs as a function of applied strain. For each of the loading geometries, several hundred LSEs were considered. To obtain an estimate of $\Omega$, Eq. (1) is fitted to each barrier-energy evolution with the constraint that the parameter $E(\tau=0)$ equals the zero-load barrier energy. Only an estimate of $\Omega$ can be obtained because Figs. 1(b)
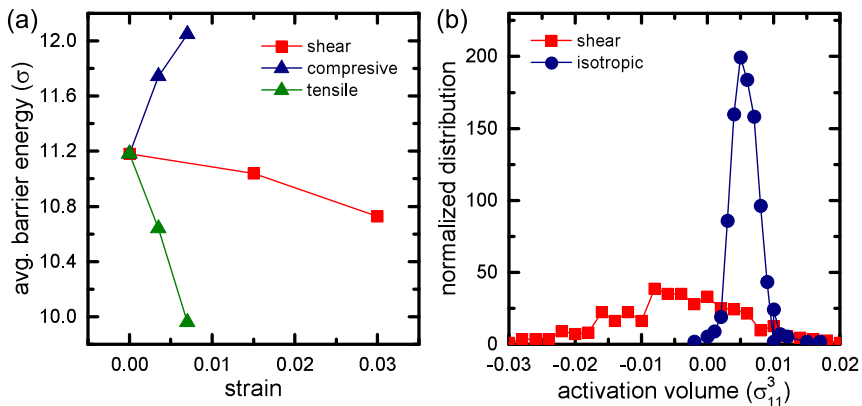

FIG. 3. (a) Mean of the barrier energies derived from the ARTn data and shown in Figs. 1 and 2, as a function of their corresponding strain magnitudes. (b) Normalized distributions of the estimated activation volume due to a pure shear and isotropic loading geometries.
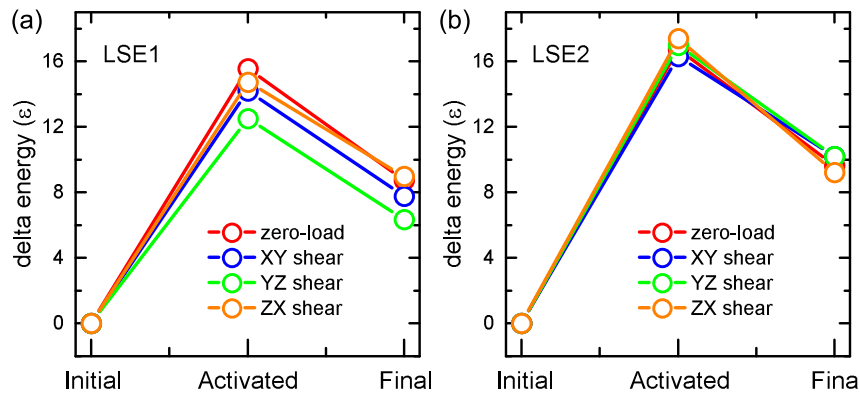

FIG. 4. Minimum-energy path of two representative LSEs shown as the energy change in going from the initial configuration to the activated and final configurations. Values are shown for the zero-load, $X Y, Y Z$, and $Z X$ shear-strain $(2 \gamma=0.03)$ configurations.

and 2(b) clearly show a nonlinear behavior that cannot be fitted well to a straight line. For isotropic strain, Fig. 3(b) shows that $\Omega$, the activation volume, is distributed with a mean of approximately $0.005 \sigma_{11}^{3}-$ a result that is compatible with the trends seen in Fig. 2(b). The distribution of $\Omega$ associated with a pure shear geometry is much broader and covers both positive and negative values, with a bias to the latter-a result that is again compatible with Figs. 1 and 3(a).

\section{Atomic visualization}

A number of LSEs are now considered in detail. To visualize the atomic-scale activity of an LSE as a function of strain and to compare it to the corresponding LSE under zero load, the affine deformation component associated with the applied shear strain is first subtracted from each configuration. Inspection of the three-dimensional nonaffine field (not shown) reveals a largely isotropic distribution of short-range atomic displacements of typical magnitude less than $\sim 0.1 \sigma_{11}$, with some local regions exhibiting larger than average displacements. Such nonaffine structures have been seen in past work [33-35].

Presently, two representative LSEs will be investigated, which are labeled as LSE1 and LSE2. Figure 4 displays the corresponding MEPs in terms of only the activated and final-state energy difference for the zero-load and three shear-strained configurations. The MEP associated with LSE1 [Fig. 4(a)] reveals a non-negligible change in barrier energy with all applied shear-strain barrier energies decreasing, demonstrating differing degrees of response of LSE barrier energies to different loading geometries, as reflected in the general changes of the LSE distributions (Fig. 1). For LSE2, the associated MEP [Fig. 4(b)] reveals a relative insensitivity to the application of shear strain, with only the $Y Z$ and $Z X$ shear-strain geometries resulting in a noticeable increase of the barrier energy.

To gain some insight into these trends, Fig. 5 plots the affinecorrected atomic displacement fields between zero-load and $X Y, Y Z$, and $Z X$ shear-strain configurations for both considered LSEs. Such displacements are visualized by vectors which point from the initial atom position to the activated atom position, and from the activated atom position to the final atom position. The balls show the initial atom position of the zero-load configuration and the color of the displacement vectors indicates the loading geometry. The figures only 


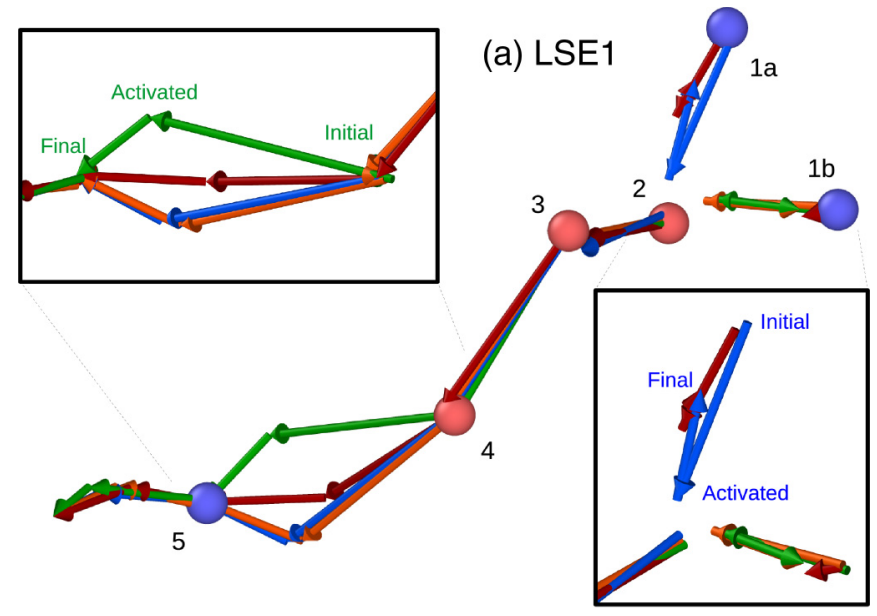

(b) LSE2

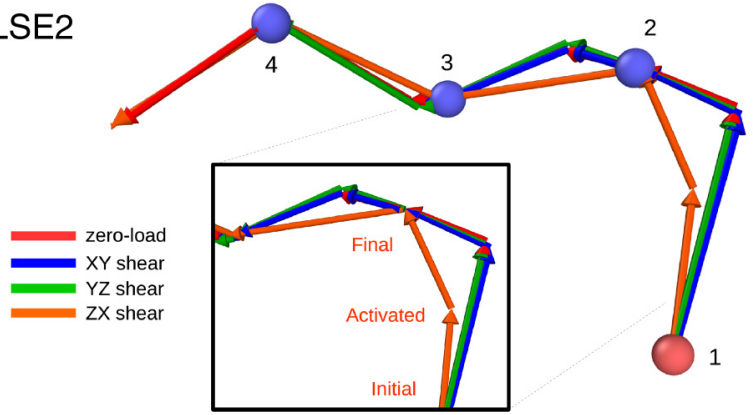

FIG. 5. Atomic displacement fields of two representative local structural excitations (LSEs): (a) LSE1 and (b) LSE2. Only the largest atomic displacements are shown. For each case, the atomic displacement vector between the initial and activated, and the activated and final, configurations is shown, with the color of the arrows reflecting the loading mode. The colored balls visualize the position of the initial zero-load configuration. Both LSEs display the common structure of sequential movement of atoms, in which one atom moves approximately a nearest-neighbor distance to replace another atom—see Ref. [20]. Graphical visualization is done using OVITO [36].

show the central LSE structure. For both LSEs, a sequence of nearest-neighbor atoms is seen to move a distance of approximately $\sigma_{11}$, demonstrating the stringlike structure of LSEs seen in Ref. [20].

Inspection of the central LSE1 structure [Fig. 5(a)] reveals differences with respect to the loading geometry. Indeed, for the $Y Z$ and $Z X$ shear-strain configurations, the atom labeled " 1 " differs from that of the zero-load and $X Y$ shear-strain configurations [see lower right inset of Fig. 5(a)]. Also, the trajectory of atom "4" [see upper left inset of Fig. 5(a)] is strongly modified by the loading mode where the saddle-point position for the $Y Z$ configuration is different from that of the $X Y$ and $Z X$ configurations. The trajectories $1 \rightarrow 2,2 \rightarrow 3$, and $5 \rightarrow 6$ (atom " 6 " not shown) are approximately within the $Y Z$ plane, whereas the trajectory $4 \rightarrow 5$ is approximately perpendicular to this plane. It is this latter trajectory that is most affected by the $Y Z$ shear strain [Fig. 4(a)].

For LSE2, inspection of the atomic displacement field [Fig. 5(b)] also reveals a stringlike structure with only the $Z X$ shear-strain central structure differing significantly from the zero-load case. This is also the shear-strain geometry for which the barrier energy increases the most [Fig. 4(b)]. Here, the combined trajectory $1 \rightarrow 2 \rightarrow 3$ is most affected [see inset of Fig. 5(b)] in which the atom position of the activated state within the trajectories $1 \rightarrow 2$ and $2 \rightarrow 3$ has changed. For this $\mathrm{LSE}$, the central structure is approximately in the $Z X$ plane.

For both LSE1 and LSE2, there exists a less local field of smaller displacements around the central structure (omitted in Fig. 5-see Ref. [20]). This surrounding field of displacements generally describes an outwards expansion of nearby atoms at the activated state, followed by a contraction as the LSE relaxes to its new local minimum (the final state). Inspection of these "accommodating" displacement fields reveals differences in their spatial extent for the three shear-strain loading geometries.

To gain quantitative insight into the origin of the barrier energy with respect to atomic displacement as a function of loading, the change in local atomic energy between the initial and activated configurations is calculated-the sum of which would give the total barrier energy. These local energies are now distinguished in terms of their corresponding atomic displacement magnitudes (between the initial and activated configurations). Figure 6 plots the cumulative barrier energy with respect to increasing displacement magnitude interval. In this figure, the horizontal axis represents an upper atomic displacement magnitude threshold, whereas the vertical axis represents the summed change in energy of these atoms satisfying this threshold - the cumulative barrier energy. The color of each data point reflects the number of atoms contributing thus far to the cumulative barrier energy. At a large enough displacement threshold, all 1728 atoms will be included and the cumulative barrier energy saturates to the actual barrier energy. As in Fig. 4, the color of the connecting line reveals the loading geometry.

Figure 4 reveals that those atoms which exhibit very small displacements contribute little to the barrier energy, with their net contribution to the barrier energy often being negative. Such atoms are generally distant from the central LSE structure and involve displacement magnitudes typical of the nonaffine deformation component $\left(<0.1 \sigma_{11}\right)$. Most of the atoms within the simulation cell are within this regime of displacement magnitude. Atoms involving displacements comparable to, but larger than, $0.1 \sigma_{11}$ tend to contribute positively to the barrier energy. Such atoms generally are in closer proximity to the LSE, generally surrounding the central structure, and contribute to about half of the total barrier energy. Those (few) central atoms with displacements in the regime of $>0.5 \sigma_{11}$ contribute to the remaining cumulative barrier energy. In the case of LSE1 [Fig. 6(a)], this contribution may either be positive or negative, whereas for LSE2 [Fig. 6(b)], it is only positive.

A number of other LSEs have been investigated in a similar manner, with particular emphasis on those with a low barrier energy. The central structure of such LSEs is also found to involve several atoms whose collective motion is chainlike. This is compatible with past ARTn work, which found little correlation between atom number and barrier energy [20]. In general, similar qualitative trends were found, with the central atomic LSE structure sometimes differing according to the shear-strain geometry, with either an increase or decrease in the corresponding barrier energy. 

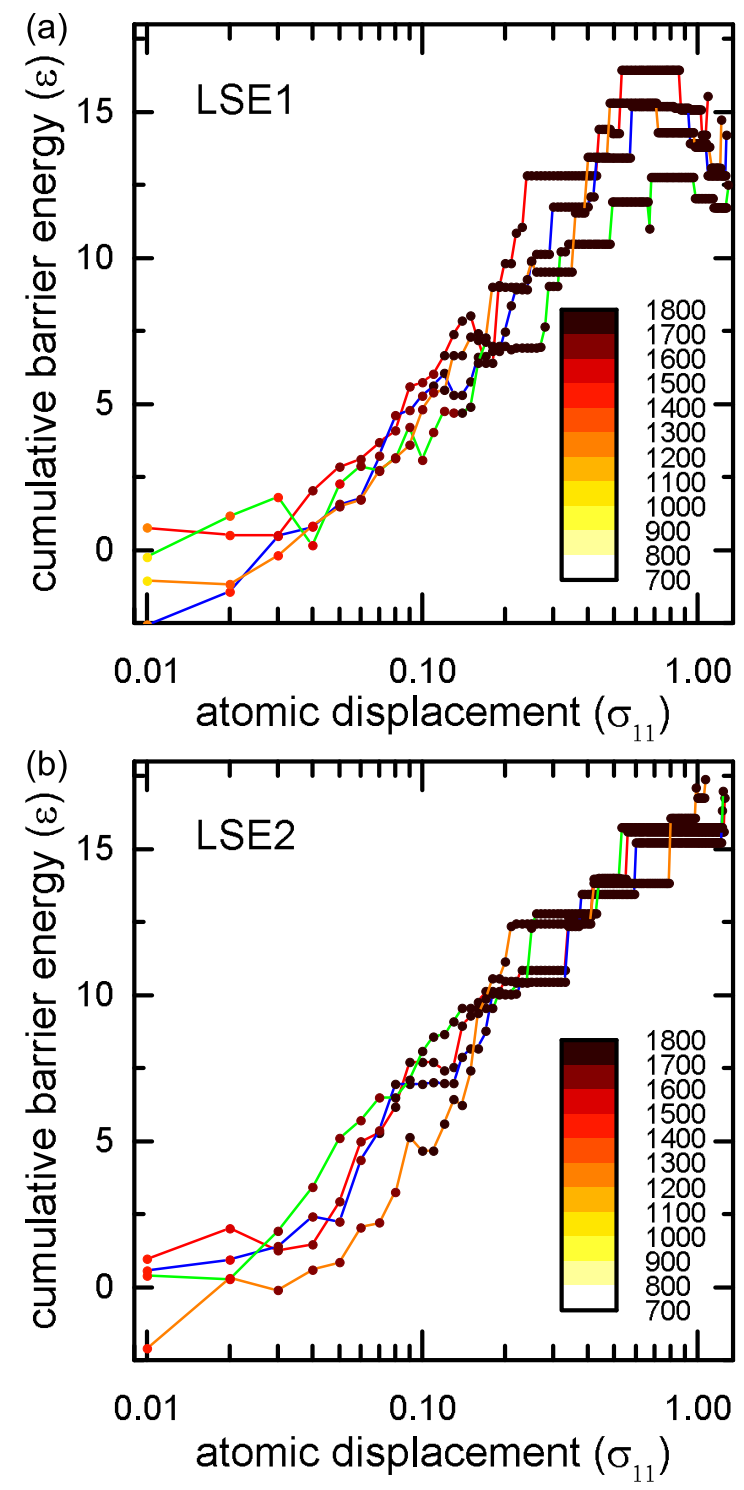

FIG. 6. For the two considered LSEs, i.e., (a) LSE1 and (b) LSE2, a plot of the cumulative barrier energy vs atomic displacement constructed by adding the change in local energy of those atoms with an atomic displacement less than the horizontal axis value. The point colors represent the number of atoms which have contributed thus far to the cumulative barrier energy, and the line colors represent the shear-strain geometries—-see the coloring scheme in Figs. 4 and 5.

\section{DISCUSSION}

The main findings of the present work are that upon an isotropic compression/tensile loading, the LSE barrier energies increase/decrease, leading to a general shift of the LSE barrierenergy distribution. On the other hand, application of a pure shear-strain results in a general broadening of the LSE barrierenergy distribution. Atomic visualization of particular LSEs under load suggests that their orientation with respect to the loading geometry could play a role in how the corresponding barrier energy is modified with respect to load. This latter result is, at best, qualitative and motivates the question of what quantitative features of a particular LSE, under zero-load
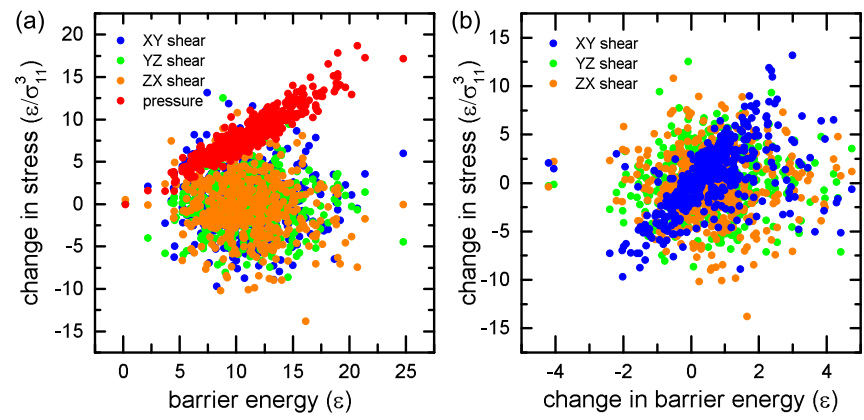

FIG. 7. (a) Scatterplot of change in internal stress between the zero-load initial and activated configurations vs barrier energy, and (b) scatterplot of change in internal shear stress between the zero-load initial and activated configurations vs the change in barrier energy as a result of an imposed $X Y$ shear strain.

conditions, influence how its barrier energy responds to an applied load.

One such signature might be the change in internal stress between the initial and activated configuration of an LSE, calculated under zero load. Figure 7(a) displays a scatterplot of such a change in stress as a function of barrier energy, in which the hydrostatic pressure and the shear stresses are shown. These quantities have been calculated using a local version of Eq. (2), which gives the local atomic stress tensor for each atom (see Ref. [20]). Inspection of the shear stresses shows little correlation with barrier energy, a trend first noticed by Rodney and Schuh [16] (see Sec. I). On the other hand, Fig. 7(a) shows a very clear linear correlation between barrier energy and the change in hydrostatic pressure (the red points in the figure). It is noted that the observed approximate linear trend of barrier energy with change in hydrostatic pressure, and the lack of a correlation with the shear components, does not result in the quadratic relation between von Mises shear and barrier energy seen in Refs. [21,22]. However, the quadratic trend seen in Refs. [21,22] is for the low-end barrier-energy regime and for barrier-energy distributions which do not tend to zero with zero barrier energy, reflecting that the atomic configurations in these works are (by design) less relaxed than the atomic configurations studied here.

The results of Fig. 7(a) gives an immediate understanding of why for a compressive load the barrier energy increases and for a tensile load it decreases [Fig. 2(b)]. Indeed, it reflects the very general result that the saddle-point configuration will always involve an expansion of the surrounding lattice to accommodate the central migrating atomic structure, which is itself in a compressive environment. In fact, approximately half of the hydrostatic pressure signature results from the surrounding lattice (here defined as displacement magnitudes comparable to $0.1 \sigma_{11}$ ), while the remainder originates directly from the central structure of the LSE. Thus, under tension/compression, the energy required for such an accommodation will be reduced/increased, resulting in a decrease/increase of the barrier energy. This is precisely the picture first proposed by Argon [3] in which there is a change in elastic energy partly within and out of the central structure, associated with the local expansion of the LSE. 
Figure 7(b) now plots the three shear-stress components already shown in Fig. 7(a) as a function of the actual change in barrier energy of the corresponding LSE, for the case of an externally applied $X Y$ shear strain (at a shear-strain magnitude of 0.03 ). For the case of the internal $X Y$ shear signature of the zero-load LSE, a linear correlation with the change in barrier energy becomes evident, whereas no such correlations are seen for the $Y Z$ and $Z X$ internal shear stresses. Again, the correlation originates from both the central LSE structure and the surrounding matrix. As in the case of isotropic strain, this result allows for a understanding of the shear-strain-dependent results shown in Fig. 1. That is, a particular LSE will have (say) a positive $X Y$ shear-stress component, and an applied shear strain (which results in a negative $X Y$ shear stress) will reduce the barrier energy - or vice versa. Given that there should be no particular bias for the "orientation" of an LSE under zero load, application of pure shear strain should only result in a broadening of the barrier-energy distribution. However, the fact that Figs. 1 and 3(a) demonstrate a small reduction in the average barrier energy with respect to an applied shear strain does suggest a more complex stress signature involving the hydrostatic stress component.

The major finding of potential-energy landscape explorations using $\mathrm{ART} n$ is that an amorphous solid admits a broad distribution of barrier energies [16-22] (Figs. 1 and 2). From the perspective of a simple thermal-activation model of plasticity characterized by a single barrier energy, this finding suggests an effective barrier energy which reflects the underlying distribution of barrier energies. Such a quantity is, however, expected to be temperature dependent, since the thermally accessible part of the distribution will shift to lower energies as the temperature decreases. Such a thermalactivation model (with only one barrier energy) works well for the high-temperature homogeneous plasticity regime of bulk metallic glasses $[1,37]$. However, because the temperature range of the high-temperature homogeneous regime of plasticity is rather narrow and accurate experimental data is difficult to obtain, a precise temperature-dependent barrier energy would be difficult to measure. It is noted that for the present binary Lennard-Jones system, the thermal fluctuation energy scale per atom is of the order of $\varepsilon$ at the glass transition, and hence the thermally accessible region of the derived distribution is at the low-barrier-energy side of the distribution peak.

As the temperature is lowered below the glass transition and the regime of the amorphous solid is entered, the temperature dependence of the yield or flow stress significantly reduces and becomes only weakly dependent on strain rate-see, for example, Refs. [1,3,11,12,37-39]—-trends that are not usually associated with thermally activated plasticity. The fact that a distribution of barrier energies exists does allow for such unusual behavior to be understood from a purely thermalactivation picture. Indeed, in the work of Refs. [40-42], it has been suggested that in this lower-temperature regime, the statistics of sampling the energy barrier distribution changes from the most probable to that of the most extreme in which only one (rare) barrier energy dominates. In these works, the external stress is assumed to affect either the first or second cumulants of the barrier-energy distribution. Indeed, Refs. [41,42] assume that the dominant effect on the barrier-energy distribution under pure shear load is to broaden the distribution - a result that is confirmed here for the model glass system studied. In fitting these models to experimental data, it is also found that for typical external loads, the effect on the distribution will be small, which is again compatible with the findings of the present work.

The activation volumes obtained in Sec. III B represent only a small fraction of an atomic volume, which is considerably smaller than what is normally seen in experiments. Indeed, experiments can give values that are typically several (or several tens of) atomic volumes (see, for example, Ref. [1]). Two aspects need to be considered when comparing the presently derived activation volumes to those extracted from experiment. First, experiments generally probe macroscopic plasticity, which at a low enough temperature involves the nucleation, propagation, and arrest of shear bands [12]. The activation volumes here represent those of individual LSEsstructural transitions that have in the past been identified as so-called $\beta$ transitions [20]—whereas macroscopic plasticity is generally believed to be mediated by $\alpha$ transitions (which are facilitated by multiple $\beta$ activity). Secondly, such activation volumes are usually derived via the assumption of a single barrier energy, which (from the discussion in the previous paragraphs) is expected to have a nontrivial temperature and stress dependence arising from both the thermal accessibility and stress dependence of the underling barrier-energy distribution. Thus the connection between an LSE's individual activation volume and that probed by experiment is not obvious. In fact, the high-temperature mean-field description of homogeneous macroplasticity developed in Ref. [42] results in a single characteristic barrier energy and activation volume, where the latter originates from the average mean-square value of the plastic shear-strain magnitude associated with the emergent $\alpha$ processes mediating plasticity within the material.

\section{CONCLUDING REMARKS}

In summary, the present work has investigated how the potential-energy landscape of a model binary Lennard-Jones glass system is modified upon application of an external stress, in particular, how the barrier-energy distribution of local structural excitations (LSEs) is modified. It is found that for isotropic compressive or tensile strain (i.e., volume change), the barrier-energy distribution shifts to higher and lower barrier energies, respectively. On the other hand, the application of pure shear strain results in a dominant broadening of the distribution. How the barrier energy of a particular LSE responds to an applied pure shear strain is found to be strongly correlated with the LSE's far-field shear-stress signature prior to loading. Such microscopic barrier-energy information as a function of loading gives quantitative insight into the energy landscape of the $\beta$ processes facilitating the $\alpha$ processes responsible for macroscopic plasticity in amorphous solids, and aids in the development of macroscopic deformation models based on a microscopic thermal-activation picture.

\section{ACKNOWLEDGMENTS}

The authors thank R. Maaß for fruitful discussions. The present work was supported by the Swiss National Science Foundation under Grant No. 200021-137871. 
[1] C. A. Schuh, T. C. Hufnagel, and U. Ramamurty, Acta Mater. 55, 4067 (2007).

[2] F. Spaepen, Acta Metall. 25, 407 (1977).

[3] A. S. Argon, Acta Metall. 27, 47 (1979).

[4] M. L. Falk and J. S. Langer, Phys. Rev. E 57, 7192 (1998).

[5] C. E. Maloney and A. Lemaitre, Phys. Rev. Lett. 93, 016001 (2004).

[6] Y. Shi and M. L. Falk, Phys. Rev. Lett. 95, 095502 (2005).

[7] M. J. Demkowicz and A. S. Argon, Phys. Rev. B 72, 245205 (2005).

[8] A. Lemaître and C. Caroli, Phys. Rev. Lett. 103, 065501 (2009).

[9] D. Klaumünzer, R. Maaß, F. H. Dalla Torre, and J. F. Löffler, Appl. Phys. Lett. 96, 061901 (2010).

[10] R. Maaß, D. Klaumüzer, and J. F. Löffler, Acta Mater. 59, 3205 (2011).

[11] R. Maaß, D. Klaumüzer, G. Villard, P. M. Derlet, and J. F. Löffler, Appl. Phys. Lett. 100, 071904 (2012).

[12] R. Maaß and J. F. Löffler, Adv. Funct. Mater. 25, 2353 (2015).

[13] G. T. Barkema and N. Mousseau, Phys. Rev. Lett. 77, 4358 (1996).

[14] N. Mousseau and G. T. Barkema, Phys. Rev. E 57, 2419 (1998).

[15] R. A. Olsen, G. J. Kroes, G. Henkelman, A. Arnaldsson, and H. Jónsson, J. Chem. Phys. 121, 9776 (2004).

[16] D. Rodney and C. A. Schuh, Phys. Rev. Lett. 102, 235503 (2009).

[17] D. Rodney and C. A. Schuh, Phys. Rev. B 80, 184203 (2009).

[18] H. Kallel, N. Mousseau, and F. Schiettekatte, Phys. Rev. Lett. 105, 045503 (2010).

[19] P. Koziatek, J-L. Barrat, P. M. Derlet, and D. Rodney, Phys. Rev. B 87, 224105 (2013).

[20] S. Swayamjyoti, J. F. Löffler, and P. M. Derlet, Phys. Rev. B 89, 224201 (2014).

[21] Y. Fan, T. Iwashita, and T. Egami, Nat. Commun. 5, 5083 (2014).

[22] Y. Fan, T. Iwashita, and T. Egami, Phys. Rev. Lett. 115, 045501 (2015).
[23] J. D. Eshelby, Proc. R. Soc. A 241, 376 (1957).

[24] G. Wahnström, Phys. Rev. A 44, 3752 (1991).

[25] A. W. Lee and S. F. Edwards, J. Phys. C 5, 1921 (1972).

[26] G. Henkelman, B. P. Uberuaga, and H. Jonsson, J. Chem. Phys. 113, 9901 (2000).

[27] G. Henkelman and H. Jonsson, J. Chem. Phys. 113, 9978 (2000).

[28] H. Jònsson, G. Mills, and K. W. Jacobsen, Classical and Quantum Dynamics in Condensed Phase Simulations, edited by B. J. Berne, G. Ciccotti, and D. F. Coker (World Scientific, Singapore, 1998), p. 385.

[29] G. Mills and H. Jonsson, Phys. Rev. Lett. 72, 1124 (1994).

[30] G. Mills, H. Jonsson, and G. K. Schenter, Surf. Sci. 324, 305 (1995).

[31] D. Caillard and J. L. Martin, Interactions between Dislocations and Small-size Obstacles Thermally Activated Mechanisms in Crystal Plasticity (Pergamon, Oxford, 2003).

[32] P. M. Derlet and R. Maaß, and J. F. Löffler, Eur. J. Phys. B 85, 148 (2012).

[33] F. Léonforte, R. Boissière, A. Tanguy, J. P. Wittmer, and J.-L. Barrat, Phys. Rev. B 72, 224206 (2005).

[34] F. Léonforte, A. Tanguy, J. P. Wittmer, and J.-L. Barrat, Phys. Rev. Lett. 97, 055501 (2006).

[35] M. Tsamados, A. Tanguy, C. Goldenberg, and J.-L. Barrat, Phys. Rev. E 80, 026112 (2009).

[36] A. Stukowski, Model. Simul. Mater. Sci. Eng. 18, 015012 (2010).

[37] J. Lu, G. Ravichandran, and W. L. Johnson, Acta Mater. 51, 3429 (2003).

[38] W. L. Johnson and K. A. Samwer, Phys. Rev. Lett. 95, 195501 (2005).

[39] E. G. Daub, D. Klaumüzer, and J. F. Löffler, Phys. Rev. E 90, 062405 (2014).

[40] P. M. Derlet and R. Maaß, Phys. Rev. B 84, 220201 (2011).

[41] P. M. Derlet and R. Maaß, Philos. Mag. 93, 4232 (2013).

[42] P. M. Derlet and R. Maaß, Philos. Mag. 94, 2776 (2014). 